\title{
THE NUMBER OF RATIONAL CURVES ON K3 SURFACES*
}

\author{
BAOSEN WU
}

\begin{abstract}
Let $X$ be a $K 3$ surface with a primitive ample divisor $H$, and let $\beta=2[H] \epsilon$ $H_{2}(X, \mathbf{Z})$. We calculate the Gromov-Witten type invariants $n_{\beta}$ by virtue of Euler numbers of some moduli spaces of stable sheaves. Eventually, it verifies Yau-Zaslow formula in the non primitive class $\beta$.
\end{abstract}

Key words. Rational curve, K3 surface, stable sheaf, Euler number

AMS subject classifications. 14N35, 14D20

Introduction. Let $X$ be a $K 3$ surface with an ample divisor $H$, and let $C \in|H|$ be a reduced curve. By adjunction formula, the arithmetic genus of $C$ is $g=\frac{1}{2} H^{2}+1$. Under the assumption that the homology class $[H] \in H_{2}(X, \mathbf{Z})$ is primitive, Yau and Zaslow [18] showed that the number of rational curves in the linear system $|H|$ is equal to the coefficient of $q^{g}$ in the series

$$
\begin{aligned}
\frac{q}{\Delta(q)}=\prod_{k>0} \frac{1}{\left(1-q^{k}\right)^{24}} & =\sum_{d \geq 0} G_{d} q^{d} \\
& =1+24 q+324 q^{2}+3200 q^{3}+25650 q^{4}+176256 q^{5}+\cdots
\end{aligned}
$$

Here a multiplicity $e(\bar{J} C)$ is assigned to each rational curve $C$ in the counting([1]).

In [5], Fantechi, Göttsche and van Straten gave an interpretation of the multiplicity $e(\bar{J} C)$. Let $M_{0,0}(X,[H])$ be the moduli space of genus zero stable maps $f: \mathbf{P}^{1} \rightarrow X$ with $f_{*}\left(\left[\mathbf{P}^{1}\right]\right)=[H] \in H_{2}(X, \mathbf{Z}) . M_{0,0}(X,[H])$ is a zero dimensional scheme which is in general nonreduced. Let $\iota: C \hookrightarrow X$ be a rational curve in the class $[H]$, and $n: \mathbf{P}^{1} \rightarrow C$ its normalization. Then $f=\iota \circ n: \mathbf{P}^{1} \rightarrow X$ is a closed point of $M_{0,0}(X,[H])$ and $e(\bar{J} C)$ is equal to the multiplicity of $M_{0,0}(X,[H])$ at $f$.

There is another formulation and generalization of Yau and Zaslow's formula by virtue of Gromov-Witten invariants. For $K 3$ surfaces, the usual genus 0 GromovWitten invariants vanish. To remedy this, one can use the notion of twistor family developed by Bryan and Leung in [2] provided that $\beta$ is a primitive class. In general, there is an algebraic geometric approach proposed by $\mathrm{Jun} \mathrm{Li} \mathrm{[11]} \mathrm{using} \mathrm{virtual} \mathrm{moduli}$ cycles. Roughly speaking, he defines Gromov-Witten type invariants $N_{g}(\beta)$ on $K 3$ surfaces by modifying the usual tangent-obstruction complex. When $\beta$ is primitive, these invariants coincide with those defined by twistor family. Geometrically, $N_{g}(\beta)$ can be thought as Gromov-Witten invariants of a one dimensional family of $K 3$ surfaces, which actually count curves in the original surface. For the rigorous definitions, see $[2],[11]$.

Bryan and Leung [2] proved a formula for $N_{g}(\beta)$ when $\beta$ is primitive. Let $n_{\beta}=$ $N_{0}(\beta)$. Then $n_{\beta}=G_{d}$ with $d=\frac{1}{2} \beta^{2}+1$. It recovers the formula of Yau and Zaslow. For a non primitive class $\beta$, the numbers $N_{g}(\beta)$ are still unknown. However, there is a conjectural formula for $N_{0}(\beta)([11])$. Using the notation $n_{\beta}$, it says

$$
n_{\beta}=\sum_{k} \frac{1}{k^{3}} G_{\frac{1}{2}\left(\frac{\beta}{k}\right)^{2}+1}
$$

\footnotetext{
*Received February 14, 2006; accepted for publication October 19, 2006.

$\dagger$ Department of Mathematics, Stanford University, Stanford, CA 94305, USA (bwu@ math.stanford.edu).
} 
where the sum runs over all integers $k>0$ such that $\frac{\beta}{k}$ is an integral homology class(see also [6]). The case $\beta=2[H]$ with $[H]$ primitive and $H^{2}=2$ was proved by Gathmann in [6].

In this paper, we will prove the following result.

Theorem 0.1. Let $X$ be a K3 surface with an ample divisor $H$. Assume $[H] \in$ $H_{2}(X, \mathbf{Z})$ is primitive. Let $\beta=2[H]$ and $g=\frac{1}{2} H^{2}+1$. Then

$$
n_{\beta}=G_{4 g-3}+\frac{1}{8} G_{g} .
$$

Now we sketch the proof of this theorem. It can be divided into two steps. First, we deform the pair $(X, H)$ to general position and then reduce the calculation of $n_{\beta}$ to $N_{\beta}$, which is the number of reduced and irreducible rational curves in $\beta$. The second step is the calculation of $N_{\beta}$. In Gathmann's approach [6], the assumption $H^{2}=2$ is essential in this step. In this paper, we will generalize the approach of Yau and Zaslow [18] according to the suggestion in [11].

Next, we describe these two steps in details.

We begin with the first step. Let $(X, H)$ be a pair of a $K 3$ surface $X$ and a primitively polarization $H$ on $X$. It is well known that two pairs $(X, H)$ and $\left(X^{\prime}, H^{\prime}\right)$ with $H^{2}=H^{\prime 2}$ are deformation equivalent. One can choose a general primitively polarized $K 3$ surface $(X, H)$, such that $\operatorname{Pic} X=\mathbf{Z} \cdot[H]$ and every rational curve in the linear system $|H|$ is nodal [3]. Moreover, using a generalization of the method in [3], one can also assume that any two rational curves in the system $|H|$ intersect transversely [4]. Now we fix such a pair $(X, H)$ once and for all. Since $n_{\beta}$ is a deformation invariant, we only need to calculate $n_{\beta}$ for such a surface.

By the enumerative interpretation of $n_{\beta}$, and follow up a similar argument as in [6], all stable maps $f: C \rightarrow X$ with $f_{*}([C])=\beta$ can be decomposed into the following three types:

1) The domain $C$ is $\mathbf{P}^{1}$, and the image $f(C) \subset X$ is a reduced and irreducible curve in the linear system $|2 H|$. We denote the number of such maps by $N_{\beta}$. The multiplicity of such $f$ is the Euler number of the compactified Jacobian of the image $f(C)$, as shown in [1].

2) The domain is a union of two $\mathbf{P}^{1}$ that intersect at one point $P$. In this case, the image is a union of two rational nodal curves that intersect at $H^{2}$ points. The image of $P$ has to be one of the intersections, hence there are $H^{2}$ such maps. Since the number of rational curves in the system $|H|$ is $G_{g}$, the total number of such maps is $\frac{1}{2} G_{g}\left(G_{g}-1\right) H^{2}$.

3) $f: C \rightarrow X$ is a double cover onto the image $f(C)$. There are two different cases:

(a) Double covers that factor through the normalization of $f(C)$, this space has dimension 2.

(b) Double covers that do not factor through the normalization. In this case, the domain must be a union of two $\mathbf{P}^{1}$, which intersect at one point $P$. The image of $P$ is a node on the image curve $f(C)$, and there is only one map for each choice of node. Note that the number of nodes on $f(C)$ is equal to the arithmetic genus $g$, so there are totally $g G_{g}$ such maps.

By Lemma 4.1 in [6], the contribution of type (3a) is $\frac{1}{8} G_{g}$. Therefore,

$$
n_{\beta}=N_{\beta}+\frac{1}{2} G_{g}\left(G_{g}-1\right) H^{2}+g G_{g}+\frac{1}{8} G_{g} .
$$


Since the first step of the proof is already known, in this paper, we will focus on the second step, namely, the calculation of the number $N_{\beta}$ of reduced and irreducible rational curves in the linear system $|2 H|$. To this end, we will work with the moduli space of sheaves on a $K 3$ surface.

Let $(X, H)$ be the pair we fixed previously. Let $\mathfrak{M}$ be the moduli scheme of stable sheaves $\mathcal{F}$ on $X$ such that $\operatorname{dim} \mathcal{F}=1, c_{1}(\mathcal{F})=2 H$ and $\chi(\mathcal{F})=1$. The Hilbert polynomial of $\mathcal{F}$ with respect to the polarization $H$ is $2 H^{2} \cdot n+1$. Since there is no strictly semistable sheaf in $\mathfrak{M}$, by [13], $\mathfrak{M}$ is a smooth projective variety, and its Euler number $e(\mathfrak{M})$ is $G_{2 H^{2}+1}([19])$. In section 1, we will construct a morphism $\Phi: \mathfrak{M} \rightarrow|2 H|$ that sends $\mathcal{F} \in \mathfrak{M}$ to its support in $|2 H|$. For $D \in|2 H|$, we denote by $\mathfrak{M}_{D}$ the fiber of $\Phi$ over $D$ with the reduced subscheme structure. When $D$ is reduced and irreducible, $\mathfrak{M}_{D}$ is the compactified Jacobian $\bar{J} D$ of $D$. In section 2 , we will show that $e\left(\mathfrak{M}_{D}\right)=0$ if $D$ has an irreducible component whose geometric genus is positive.

Therefore only divisors with rational components contribute to the Euler number $e(\mathfrak{M})$. Since $H$ is primitive, we have three types of these divisors in the linear system $|2 H|$.

1) $D=C, C$ is a rational curve in homology class $\beta(=2[H])$. In this case, $\mathfrak{M}_{D} \cong \bar{J} D$. The number of such divisors $D$, counted with multiplicity $e(\bar{J} D)$, is equal to $N_{\beta}$.

2) $D=C_{1}+C_{2}$, where $C_{1}$ and $C_{2}$ are different rational nodal curves. In this case, both $C_{i}$ are contained in the linear system $|H|$. There are totally $\frac{1}{2} G_{g}\left(G_{g}-1\right)$ divisors of this type. We will show that $e\left(\mathfrak{M}_{D}\right)=H^{2}$ in section 3 .

3) $D=2 C_{0}$, where $C_{0}$ is a rational nodal curve and contained in $|H|$. The number of such divisors is $G_{g}$. In the last two sections we will prove $e\left(\mathfrak{M}_{D}\right)=g$, which is equal to the number of nodes of $C_{0}$.

Since $e(\mathfrak{M})=\sum e\left(\mathfrak{M}_{D}\right)$, where the sum runs over all divisors $D$ with rational components, we get

$$
\begin{aligned}
N_{\beta} & =e(\mathfrak{M})-\frac{1}{2} G_{g}\left(G_{g}-1\right) H^{2}-g G_{g} \\
& =G_{4 g-3}-\frac{1}{2} G_{g}\left(G_{g}-1\right) H^{2}-g G_{g} .
\end{aligned}
$$

Together with (1), we prove

$$
n_{\beta}=G_{4 g-3}+\frac{1}{8} G_{g} .
$$

Recently, J. Li and the author [12] proved the conjectured formula for non primitive class $\beta=n[H]$ with $n<6$, under the assumption that the transversality of rational curves still holds.

I am most grateful to Jun Li, from whom I learned moduli spaces of sheaves and Gromov-Witten invariants. During the preparation of this paper, his constant encouragement and discussions are invaluable. After finishing the manuscript, the author is informed that J.Lee and N.C.Leung [9] proved the same result using degeneration method and also counted genus 1 curves in $K 3$ surfaces [10].

1. Decomposition of the moduli scheme $\mathfrak{M}$. We start with some definitions and notations $([15],[8])$.

Let $X$ be a complex projective scheme with an ample line bundle $\mathcal{O}(1)$. For a coherent sheaf $\mathcal{E}$ of $\mathcal{O}_{X}$-module, the Hilbert polynomial $p(\mathcal{E}, n)$ of $\mathcal{E}$ is defined as

$$
p(\mathcal{E}, n)=\operatorname{dim} H^{0}(X, \mathcal{E}(n)), n \gg 0 .
$$


The dimension of the support of $\mathcal{E}$ is equal to the degree of $p(\mathcal{E}, n)$. A coherent sheaf $\mathcal{E}$ is pure of dimension $d$ if for any nonzero coherent subsheaf $\mathcal{F} \subset \mathcal{E}, \operatorname{dim} \mathcal{F}=d$.

The Hilbert polynomial $p(\mathcal{E}, n)$ can be written as

$$
p(\mathcal{E}, n)=\frac{a_{0}}{d !} n^{d}+\frac{a_{1}}{(d-1) !} n^{d-1}+\cdots
$$

with integral coefficients $a_{i}=a_{i}(\mathcal{E})$. We define the slope of $\mathcal{E}$ to be

$$
\mu(\mathcal{E})=a_{0}\left(\mathcal{O}_{X}\right) \frac{a_{1}(\mathcal{E})}{a_{0}(\mathcal{E})}-a_{1}\left(\mathcal{O}_{X}\right) .
$$

Definition 1.1. A coherent sheaf $\mathcal{E}$ is stable (resp. semistable) if it is pure, and if for any nonzero proper subsheaf $\mathcal{F} \subset \mathcal{E}$, there exists an $N$, such that for $n>N$,

$$
\frac{p(\mathcal{F}, n)}{a_{0}(\mathcal{F})}<\frac{p(\mathcal{E}, n)}{a_{0}(\mathcal{E})} \quad(\text { resp. } \leq)
$$

Definition 1.2. A coherent sheaf $\mathcal{E}$ is $\mu$-stable (resp. $\mu$-semistable) if it is pure, and if for any nonzero proper subsheaf $\mathcal{F} \subset \mathcal{E}$,

$$
\mu(\mathcal{F})<\mu(\mathcal{E}) \quad(\text { resp. } \leq) .
$$

THEOREM 1.3. [15]Let $X$ be a complex projective scheme with an ample line bundle $\mathcal{O}(1)$. There is a projective coarse moduli scheme whose closed points represent the $S$-equivalence classes of semistable sheaves with Hilbert polynomial $P(n)$.

Let $X$ be a K3 surface with an ample line bundle $H$. By Riemann-Roch theorem, the Hilbert polynomial of a torsion free sheaf $\mathcal{E}$ is

$$
p(\mathcal{E}, n)=\frac{r}{2} H^{2} n^{2}+\left(c_{1} \cdot H\right) n+r \chi\left(\mathcal{O}_{X}\right)+\frac{1}{2}\left(c_{1}^{2}-2 c_{2}\right),
$$

where $r$ is the rank of $\mathcal{E}$ and $c_{i}=c_{i}(\mathcal{E})$. Let $\mathcal{F}$ be a pure sheaf of dimension 1 on $X$. By a locally free resolution, one can verify that the Hilbert polynomial of $\mathcal{F}$ is

$$
p(\mathcal{F}, n)=\left(c_{1}(\mathcal{F}) \cdot H\right) n+\frac{1}{2}\left(c_{1}^{2}(\mathcal{F})-2 c_{2}(\mathcal{F})\right) .
$$

It is clear that for such sheaves the notion of stability and $\mu$-stability coincide.

From now on, we fix a pair $(X, H)$ of a $K 3$ surface $X$ and a polarization $H$ of $X$, such that

1) $\operatorname{Pic} X=\mathbf{Z} \cdot[H]$;

2) every rational curve in $|H|$ is nodal; and

3 ) any two distinct rational curves in $|H|$ intersect transversely.

We let $\beta=2[H] \in H_{2}(X, \mathbf{Z})$. Our immediate goal is to calculate $N_{\beta}$, the number of reduced and irreducible rational curves in $|2 H|$ counted with multiplicity. To this end, we consider the moduli scheme $\mathfrak{M}$ of stable sheaves $\mathcal{F}$ of $\mathcal{O}_{X}$-modules that satisfy $\operatorname{dim} \mathcal{F}=1, c_{1}(\mathcal{F})=\beta$ and $\chi(\mathcal{F})=1$.

TheOREm 1.4. [19] $\mathfrak{M}$ is a smooth projective variety. The Euler number e $(\mathfrak{M})$ is $G_{2 H^{2}+1}$. 
Next we define the morphism $\Phi: \mathfrak{M} \rightarrow|2 H|$ mentioned earlier.

Let $\mathcal{F}$ be a sheaf in $\mathfrak{M}$. Since $\mathcal{F}$ is pure of dimension 1 , it admits a length 1 locally free resolution

$$
0 \longrightarrow \mathcal{E}_{1} \stackrel{f}{\longrightarrow} \mathcal{E}_{0} \longrightarrow \mathcal{F} \longrightarrow 0,
$$

with $r\left(\mathcal{E}_{1}\right)=r\left(\mathcal{E}_{0}\right)$. The homomorphism $f: \mathcal{E}_{1} \rightarrow \mathcal{E}_{0}$ induces a homomorphism $\wedge^{r} f: \wedge^{r} \mathcal{E}_{1} \rightarrow \wedge^{r} \mathcal{E}_{0}$, and a nonzero global section $\left.s \in H^{0}\left(\wedge^{r} \mathcal{E}_{1}\right)^{-1} \otimes\left(\wedge^{r} \mathcal{E}_{0}\right)\right)$, that defines an effective divisor $D=s^{-1}(0)$ on $X$. Since $\left(\wedge^{r} \mathcal{E}_{1}\right)^{-1} \otimes\left(\wedge^{r} \mathcal{E}_{0}\right)=c_{1}(\mathcal{F})=2 H$, $D$ is contained in the linear system $|2 H|$. The assignment $\mathcal{F} \rightarrow D$ defines a morphism $\Phi: \mathfrak{M} \rightarrow|2 H|$.

We now give a specific decomposition of the projective space $|2 H|$ according to the topological type of $D \in|2 H|$.

We let $\mathcal{W}_{1}$ be the set of divisors $D$ which is reduced and irreducible. The arithmetic genus of $D$ is $p_{a}(D)=2 H^{2}+1$, which is an invariant for all $D \in \mathcal{W}_{1}$. We further stratify $\mathcal{W}_{1}$ according to the geometric genus of curves, $\mathcal{W}_{1}=\sqcup_{k} \mathcal{W}_{1}^{k}$, where $\mathcal{W}_{1}^{k}$ consists of those $D$ that have geometric genus $k$. Clearly $\sqcup_{k \leq a} \mathcal{W}_{1}^{k}$ is closed in $\mathcal{W}_{1}$. Let $\mathcal{W}_{2}$ be the stratum of divisors $D=C_{1}+C_{2}$ with $C_{1} \neq C_{2}$ and $C_{i} \in|H|$. Without loss of generality, we can assume $p_{g}\left(C_{1}\right) \leq p_{g}\left(C_{2}\right)$. For $a \leq b$, we let $\mathcal{W}_{2}^{a, b} \subset \mathcal{W}_{2}$ be the subset of divisors $D$ with $p_{g}\left(C_{1}\right)=a$ and $p_{g}\left(C_{2}\right)=b$. Then $\mathcal{W}_{2}=\sqcup \mathcal{W}_{2}^{a, b}$. Let $\mathcal{W}_{3}$ be the subset of divisors $D=2 C_{0}$ with $C_{0} \in|H|$. Similarly, $\mathcal{W}_{3}=\sqcup_{k} \mathcal{W}_{3}^{k}$, where $\mathcal{W}_{3}^{k}$ consists of $D=2 C_{0}$ with $p_{g}\left(C_{0}\right)=k$.

Put together,

$$
|2 H|=\left(\sqcup_{k} \mathcal{W}_{1}^{k}\right) \bigsqcup\left(\sqcup_{a \leq b} \mathcal{W}_{2}^{a, b}\right) \bigsqcup\left(\sqcup_{k} \mathcal{W}_{3}^{k}\right)
$$

This induces a decomposition on $\mathfrak{M}$,

$$
\mathfrak{M}=\left(\sqcup_{k} \Phi^{-1}\left(\mathcal{W}_{1}^{k}\right)\right) \bigsqcup\left(\sqcup \Phi^{-1}\left(\mathcal{W}_{2}^{a, b}\right)\right) \bigsqcup\left(\sqcup_{k} \Phi^{-1}\left(\mathcal{W}_{3}^{k}\right)\right) .
$$

Now we state a general fact on the Euler number of varieties.

Let $Z$ be a complex variety. Let $Z=\sqcup Z_{i}$ be a decomposition into locally closed subset $Z_{i}$. Then the Euler number $e(Z)=\sum e\left(Z_{i}\right)$.

Apply this to the decomposition of $\mathfrak{M}$, we have

$$
\begin{aligned}
e(\mathfrak{M}) & =e\left(\Phi^{-1}\left(\mathcal{W}_{1}\right)\right)+e\left(\Phi^{-1}\left(\mathcal{W}_{2}\right)\right)+e\left(\Phi^{-1}\left(\mathcal{W}_{3}\right)\right) \\
& =\sum_{k} e\left(\Phi^{-1}\left(\mathcal{W}_{1}^{k}\right)\right)+\sum_{a \leq b} e\left(\Phi^{-1}\left(\mathcal{W}_{2}^{a, b}\right)\right)+\sum_{k} e\left(\Phi^{-1}\left(\mathcal{W}_{3}^{k}\right)\right) .
\end{aligned}
$$

Proposition 1.5. [1] Let $h: Y \rightarrow Z$ be a surjective morphism between complex algebraic varieties. Suppose that $e\left(h^{-1}(z)\right)=0$ for every closed point $z \in Z$. Then $e(Y)=0$.

The following proposition will be proved in the next section.

Proposition 1.6. Suppose $D$ is a divisor that has one irreducible component whose geometric genus is positive, then $e\left(\mathfrak{M}_{D}\right)=0$.

Combine these results, we have

$$
e(\mathfrak{M})=e\left(\Phi^{-1}\left(\mathcal{W}_{1}^{0}\right)\right)+e\left(\Phi^{-1}\left(\mathcal{W}_{2}^{0,0}\right)\right)+e\left(\Phi^{-1}\left(\mathcal{W}_{3}^{0}\right)\right) .
$$


Because $e\left(\Phi^{-1}\left(\mathcal{W}_{1}^{0}\right)\right)$ is equal to $N_{\beta}$, and $e(\mathfrak{M})=G_{2 H^{2}+1}$, To calculate $N_{\beta}$, it suffices to find the Euler numbers $e\left(\Phi^{-1}\left(\mathcal{W}_{2}^{0,0}\right)\right)$ and $e\left(\Phi^{-1}\left(\mathcal{W}_{3}^{0}\right)\right)$. The number $e\left(\Phi^{-1}\left(\mathcal{W}_{2}^{0,0}\right)\right)$ is essentially known, which is equal to $\frac{1}{2} G_{g}\left(G_{g}-1\right) H^{2}$ as will be shown in section 3 . The main body of the remainder of the paper is to show that $e\left(\Phi^{-1}\left(\mathcal{W}_{3}^{0}\right)\right)=g G_{g}$. Therefore,

$$
N_{\beta}=G_{2 H^{2}+1}-\frac{1}{2} G_{g}\left(G_{g}-1\right) H^{2}-g G_{g} .
$$

Apply equality (1) in the introduction, we obtain the formula in the main theorem.

2. Proof of Proposition 1.6. We state a basic fact about the Euler number of a variety. Let $X$ be a quasi-projective variety. If there exists a finite group action on $X$ which is free of fixed point, then $e(X)$ is divisible by the order of this group. Therefore, if for any positive integer $N$, there is a finite group $G_{N}$ whose order is greater than $N$, and a free $G_{N}$ action on $X$, then $e(X)$ is zero.

If $D$ is a reduced and irreducible curve, then $\mathfrak{M}_{D} \cong \bar{J} D$. Since the geometric genus of $D$ is positive, $e(\bar{J} D)=0$ (see[1]). Now if $D=C_{1}+C_{2}$ with $C_{i} \in|H|$ and by assumption the geometric genus $p_{g}\left(C_{2}\right)>0$. From the restriction homomorphism $\alpha: \operatorname{Pic} D \rightarrow \operatorname{Pic} C_{2}$, we can choose a subgroup $G \subset \operatorname{Pic} D$, such that for $\mathcal{L} \in G$, $\left.\mathcal{L}\right|_{C_{1}} \cong \mathcal{O}_{C_{1}}$ and $\alpha(\mathcal{L})=\left.\mathcal{L}\right|_{C_{2}}$ is trivial if and only if $\mathcal{L}$ is trivial. Next we show that the $G$-action on $\mathfrak{M}_{D}$ defined by tensorization is free, i.e., for any sheaf $\mathcal{F} \in \mathfrak{M}_{D}$ and $\mathcal{L} \in G, \mathcal{F} \otimes \mathcal{L} \cong \mathcal{F}$ if and only if $\mathcal{L}$ is trivial. To this end, suppose $\mathcal{F} \otimes \mathcal{L} \cong \mathcal{F}$ for some $\mathcal{F}$ and $\mathcal{L}$. Let $\mathcal{F}_{2}$ be the torsion free part of the restriction $\left.\mathcal{F}\right|_{C_{2}}$. We obtain $\mathcal{F}_{2} \otimes \alpha(\mathcal{L}) \cong \mathcal{F}_{2}$ and therefore $\alpha(\mathcal{L})$ is trivial by the same argument as in case 1 . Finally, it implies $\mathcal{L}$ is trivial by the choice of the subgroup $G$. Finally, $D=2 C_{0}$ is a divisor whose associated subscheme is a nonreduced curve $C$, and a closed point in $\mathfrak{M}_{D}$ is a sheaf of $\mathcal{O}_{C}$-modules. To prove this case, we first recall some facts on nonreduced curves.

Let $\mathcal{F}$ be a sheaf of $\mathcal{O}_{X}$-modules. An infinitesimal extension([7], Exer II 8.7) of $X$ by $\mathcal{F}$ is a scheme $X^{\prime}$, with an ideal sheaf $\mathcal{I}$, such that $\mathcal{I}^{2}=0$ and $\left(X^{\prime}, \mathcal{O}_{X^{\prime}} / \mathcal{I}\right) \cong$ $\left(X, \mathcal{O}_{X}\right)$ and such that $\mathcal{I}$ with the induced structure of $\mathcal{O}_{X}$-module is isomorphic to the given sheaf $\mathcal{F}$. Let $S$ be a smooth projective surface, and $C_{0} \subset S$ be a reduced and irreducible curve. There is an associated closed subscheme $C \subset S$ to the divisor $2 C_{0}$. In fact, $C$ is an infinitesimal extension of $C_{0}$ by $\mathcal{I}=\left.\mathcal{O}_{S}\left(-C_{0}\right)\right|_{C_{0}}$.

Next we discuss the Picard group of $C$ ([7], Exer III 4.6). From the exact sequence of sheaves of abelian groups

$$
0 \longrightarrow \mathcal{I} \longrightarrow \mathcal{O}_{C}^{*} \longrightarrow \mathcal{O}_{C_{0}}^{*} \longrightarrow 0
$$

there is an induced exact sequence

$$
0 \longrightarrow H^{1}(C, \mathcal{I}) \longrightarrow \operatorname{Pic} C \longrightarrow \operatorname{Pic} C_{0} \longrightarrow 0 .
$$

Notice that $H^{1}(C, \mathcal{I})$ is a vector space and hence an injective $\mathbf{Z}$-module, it implies that Pic $C \cong \operatorname{Pic} C_{0} \oplus H^{1}(C, \mathcal{I})$ as groups. For $\mathcal{L} \in \operatorname{Pic} C$, we let $\mathcal{L}_{0} \in \operatorname{Pic} C_{0}$ be the restriction of $\mathcal{L}$ to $C_{0}$.

Now we continue the proof. Let $\pi: \tilde{C}_{0} \rightarrow C_{0}$ be the normalization of $C_{0}$. Then $\operatorname{Pic}^{0} C_{0} \cong \operatorname{Pic}^{0} \tilde{C}_{0} \oplus A$, where $A$ is an affine commutative group. Since the genus of $\tilde{C}_{0}$ is positive, $\operatorname{Pic}^{0} \tilde{C}_{0}$ is nontrivial. For any odd prime $p$, we can choose an order $p$ subgroup $G \subset \operatorname{Pic}^{0} C$, such that for $\mathcal{L} \in G, \mathcal{L}$ is trivial if and only if $\tilde{\mathcal{L}}=\pi^{*} \mathcal{L}_{0}$ is 
trivial. There is a $G$-action on $\mathfrak{M}_{D}$ defined by tensorization. Next we show that this group action is free and therefore $e\left(\mathfrak{M}_{D}\right)=0$.

Suppose $\mathcal{L} \otimes \mathcal{E} \cong \mathcal{E}$ for some sheaf $\mathcal{E} \in \mathfrak{M}_{D}$ and $\mathcal{L} \in G$. Restrict to $C_{0}$ and let $\mathcal{E}_{0}$ be the torsion free part of $\mathcal{E} \otimes \mathcal{O}_{C_{0}}$, we get $\mathcal{L}_{0} \otimes \mathcal{E}_{0} \cong \mathcal{E}_{0}$.

1) If $\mathcal{I E} \neq 0, \mathcal{E}_{0}$ is a rank 1 torsion free sheaf on $C_{0}$. Using the same argument as in case 1 , we obtain $\mathcal{L}_{0} \cong \mathcal{O}_{C_{0}}$, and hence $\mathcal{L} \cong \mathcal{O}_{C}$, i.e., the group action is free.

2) If $\mathcal{I E}=0, \mathcal{E}_{0}$ is a rank 2 torsion free sheaf on $C_{0}$. Let $\tilde{\mathcal{E}}_{0}$ be the torsion free part of $\pi^{*} \mathcal{E}_{0}$. Then we have $\tilde{\mathcal{L}}_{0} \otimes \tilde{\mathcal{E}}_{0} \cong \tilde{\mathcal{E}}_{0}$. Take top wedge on both sides, we get $\tilde{\mathcal{L}}_{0}^{\otimes 2} \otimes \wedge^{2} \tilde{\mathcal{E}}_{0} \cong \wedge^{2} \tilde{\mathcal{E}}_{0}$. Since $\wedge^{2} \tilde{\mathcal{E}}_{0}$ is invertible, $\tilde{\mathcal{L}}_{0}^{\otimes 2} \cong \mathcal{O}_{\tilde{C}_{0}}$. Note that $\mathcal{L} \in G$ and $G$ has odd prime order $p$, it implies that $\mathcal{L} \cong \mathcal{O}_{C}$. Hence the group action is free.

3. Calculation of $e\left(\Phi^{-1}\left(\mathcal{W}_{2}^{0,0}\right)\right)$. Recall that $\mathcal{W}_{2}^{0,0}$ is a finite set of divisors $D=C_{1}+C_{2}$ with $C_{1}, C_{2} \in|H|$ being rational nodal curves and intersect transversally, $\Phi^{-1}\left(\mathcal{W}_{2}^{0,0}\right)=\sqcup \mathfrak{M}_{D_{i}}$ with $D_{i} \in \mathcal{W}_{2}^{0,0}$. We will calculate $e\left(\mathfrak{M}_{D}\right)$ for $D \in \mathcal{W}_{2}^{0,0}$ and then the Euler number $e\left(\Phi^{-1}\left(\mathcal{W}_{2}^{0,0}\right)\right)$ follows.

A closed point in $\mathfrak{M}_{D}$ is a stable sheaf $\mathcal{E}$ of $\mathcal{O}_{D}$-modules, such that the restrictions $\left.\mathcal{E}\right|_{C_{i}}$ are rank 1 sheaves of $\mathcal{O}_{C_{i}}$-modules respectively. Let $x_{1}, x_{2}, \cdots, x_{s}$ be a list of intersections of $C_{1}$ and $C_{2}$. Then $s=H^{2}>0$. Since $\mathcal{E}$ is stable, there is at least one point $x_{k}$, so that the stalk $\mathcal{E}_{x_{k}}$ is isomorphic to $\mathcal{O}_{x_{k}}$. For otherwise, $\mathcal{E}$ is the direct image of some sheaf on the disjoint union of $C_{1}$ and $C_{2}$, which violates the stability of $\mathcal{E}$.

We let $S_{i j} \subset \mathfrak{M}_{D}$ be the subset of stable sheaves $\mathcal{E}$ such that $\mathcal{E}_{x_{i}} \cong \mathcal{O}_{x_{i}}$ and $\mathcal{E}_{x_{j}} \cong \mathcal{O}_{x_{j}}$ for two intersection points $x_{i}$ and $x_{j}$. We can find a subgroup $G \subset \operatorname{Pic} D$ coming from the gluing of $\mathcal{O}_{C_{1}}$ and $\mathcal{O}_{C_{2}}$ at $x_{i}$ and $x_{j} . G \cong \mathbf{C}^{*}$. Now follow a similar argument as in the previous section, the $G$-action on $S_{i j}$ is free. Therefore, the contribution to the Euler number $e\left(\mathfrak{M}_{D}\right)$ come from stable sheaves $\mathcal{E}$ whose stalks are not $\mathcal{O}$ at all nodes but one intersection point. Since both $C_{i}$ are rational curves, there is only one such stable sheaf corresponds to an intersection point. We have

Proposition 3.1. Let $D$ be a divisor in the set $\mathcal{W}_{2}^{0,0}$. Then $e\left(\mathfrak{M}_{D}\right)=H^{2}$.

Since the number of rational curves in $|H|$ is $G_{g}, \mathcal{W}_{2}^{0,0}$ is a finite set with cardinality $\frac{1}{2} G_{g}\left(G_{g}-1\right)$.

Corollary 3.2. $e\left(\Phi^{-1}\left(\mathcal{W}_{2}^{0,0}\right)\right)=\frac{1}{2} G_{g}\left(G_{g}-1\right) H^{2}$.

4. Calculation of $e\left(\Phi^{-1}\left(\mathcal{W}_{3}^{0}\right)\right)$, Part I. In the remainder of this paper, we will calculate the Euler number $e\left(\Phi^{-1}\left(\mathcal{W}_{3}^{0}\right)\right)$. Remember that $\mathcal{W}_{3}^{0}$ is a finite set of divisors $D=2 C_{0}$ with $C_{0} \in|H|$ being rational nodal curves, there is a decomposition $\Phi^{-1}\left(\mathcal{W}_{3}^{0}\right)=\sqcup \mathfrak{M}_{D_{i}}$. It suffices to calculate $e\left(\mathfrak{M}_{D}\right)$ for $D \in \mathcal{W}_{3}^{0}$.

Recall that for every effective divisor, there is an associated subscheme. Let $C$ be the nonreduced curve associated to $D=2 C_{0}$. Then every closed point in $\mathfrak{M}_{D}$ corresponds to a stable sheaf $\mathcal{E}$ of $\mathcal{O}_{C}$-modules, such that the Hilbert polynomial $P_{\mathcal{E}}(n)$ is $2 H^{2}+1$.

There are two kinds of these sheaves. A sheaf $\mathcal{E}$ in the first type satisfies $\mathcal{I E}=0$, where $\mathcal{I} \subset \mathcal{O}_{C}$ is the nilpotent ideal sheaf. That is to say, $\mathcal{E}$ is a rank 2 sheaf on $C_{0}$, the reduced part of $C$. Let $\mathfrak{M}_{D}^{1} \subset \mathfrak{M}_{D}$ be the subset of sheaves of this type. The second type consists of sheaves $\mathcal{E}$ satisfy $\mathcal{I E} \neq 0$. It is direct to verify that for sheaves of this type, $\mathcal{E}_{\eta} \cong \mathcal{O}_{\eta}$ with $\eta$ the generic point of $C$. Let $\mathfrak{M}_{D}^{2}$ be the subset of sheaves of the second type. Then $e\left(\mathfrak{M}_{D}\right)=e\left(\mathfrak{M}_{D}^{1}\right)+e\left(\mathfrak{M}_{D}^{2}\right)$.

In this section, we calculate $e\left(\mathfrak{M}_{D}^{1}\right)$. The discussion of $\mathfrak{M}_{D}^{2}$ is left to the next section. The result has been obtained by T. Teodorescu in his $\mathrm{PhD}$ thesis [16] which 
deals with a more general problem. It is also proved in [17] independently. Now we use a slightly different approach.

We recall some standard facts about sheaves on a nodal curve. Since we will not talk about nonreduced curves in the next part of this section, we use $C$, instead of $C_{0}$, to denote a nodal curve. We always work on the complex topology.

Let $C$ be a projective curve with $n$ ordinary nodes $x_{1}, x_{2}, \cdots, x_{n}$ as singularities, and let $\pi: \tilde{C} \rightarrow C$ be the normalization of $C$. A torsion free sheaf $\mathcal{E}$ is locally free away from the nodes. It has the following nice local structure at each node $x_{i} \in C([14])$

$$
\mathcal{E}_{x_{i}} \cong \mathcal{O}_{x_{i}}^{\oplus a_{i}} \oplus m_{x_{i}}^{\oplus\left(r-a_{i}\right)},
$$

where $m_{x_{i}} \subset \mathcal{O}_{x_{i}}$ is the maximal ideal, and $r$ is the rank of $\mathcal{E}$. Let $\hat{\pi}: \hat{C} \rightarrow C$ be a partial normalization of $C$ at one node $x$. Then there exists a torsion free sheaf $\mathcal{F}$ on $\hat{C}$ such that $\mathcal{E} \cong \hat{\pi}_{*} \mathcal{F}$ if and only if $\mathcal{E}_{x} \cong m_{x}^{\oplus r}$.

Let $r \geq 1$ be an integer and choose $n$ such that $(r, n)=1$. There is a smooth projective variety $\mathfrak{M}_{C}(r, n)$ whose closed points correspond to isomorphism classes of stable $\mathcal{O}_{C}$-modules $\mathcal{E}$, such that $r(\mathcal{E})=r$ and $\chi(\mathcal{E})=n$.

Next we introduce the notion of admissible quotients. It will be used to determine whether two torsion free sheaves $\mathcal{E}_{1}, \mathcal{E}_{2}$ are isomorphic. Let $\mathcal{E}$ be a torsion free sheaf and $\left(\pi^{*} \mathcal{E}\right)^{\sharp}$ be the torsion free part of $\pi^{*} \mathcal{E}$. There is a canonical exact sequence

$$
0 \longrightarrow \mathcal{E} \longrightarrow \pi_{*}\left(\pi^{*} \mathcal{E}\right)^{\sharp} \longrightarrow \mathcal{T} \longrightarrow 0,
$$

where $\mathcal{T} \cong \oplus \mathbf{C}_{x_{i}}^{\oplus a_{i}}$ is a skyscraper sheaf supported at the nodes.

Definition 4.1. Let $\mathcal{V}$ be a rank $r$ locally free sheaf on $\tilde{C}$, and $\mathcal{Q}=\oplus \mathbf{C}_{x_{i}}^{\oplus a_{i}}$ be a skyscraper sheaf supported at the set of nodes on $C$. Let $\rho: \pi_{*} \mathcal{V} \rightarrow \mathcal{Q}$ be a surjective morphism and $\mathcal{E}$ be the kernel of $\rho . \rho$ is said to be an admissible quotient if there is a commutative diagram

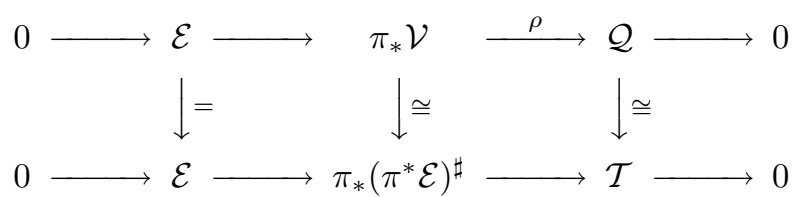

where the second row is the canonical exact sequence.

Let $p_{i}, q_{i} \in \tilde{C}$ be the inverse images of the node $x_{i}$. Then $\left(\pi_{*} \mathcal{V}\right)_{x_{i}}=\mathcal{V}_{p_{i}} \oplus \mathcal{V}_{q_{i}}$. The homomorphism $\rho$ is given by $\rho_{x_{i}}: \mathcal{V}_{p_{i}} \oplus \mathcal{V}_{q_{i}} \rightarrow \mathcal{Q}_{x_{i}}$. Let $\iota_{i}^{1}: \mathcal{V}_{p_{i}} \rightarrow \mathcal{V}_{p_{i}} \oplus \mathcal{V}_{q_{i}}$ and $\iota_{i}^{2}: \mathcal{V}_{q_{i}} \rightarrow \mathcal{V}_{p_{i}} \oplus \mathcal{V}_{q_{i}}$ be the natural injections. By the definition of admissible quotients, $\rho_{x_{i}}^{k}=\rho_{x_{i}} \circ \iota_{i}^{k}$ are both surjective. Conversely, we have

Proposition 4.2. Let $\rho: \pi_{*} \mathcal{V} \rightarrow \mathcal{Q}$ be a quotient such that $\rho_{x_{i}}^{k}$ defined above are surjective for all $i=1,2 \cdots, n$ and $k=1,2$. Then $\rho$ is an admissible quotient.

Proof. Let $\mathcal{E}$ be the kernel of $\rho$. Apply the functor $\pi^{*}$ to the exact sequence

$$
0 \longrightarrow \mathcal{E} \longrightarrow \pi_{*} \mathcal{V} \stackrel{\rho}{\longrightarrow} \mathcal{Q} \longrightarrow 0,
$$

we get

$$
\pi^{*} \mathcal{E} \stackrel{\psi_{1}}{\longrightarrow} \pi^{*}\left(\pi_{*} \mathcal{V}\right) \stackrel{\psi_{2}}{\longrightarrow} \pi^{*} \mathcal{Q} \longrightarrow 0
$$


Since $\rho_{x_{i}}^{k}$ are surjective, the restriction of $\psi_{2}$ on the torsion part $\mathcal{T}^{\prime} \subset \pi^{*}\left(\pi_{*} \mathcal{V}\right)$ is surjective, i.e. $\psi_{2}\left(\mathcal{T}^{\prime}\right)=\pi^{*} \mathcal{Q}$. It implies that the homomorphism $\pi^{*} \mathcal{E} \rightarrow$ $\left(\pi^{*}\left(\pi_{*} \mathcal{V}\right)\right)^{\sharp}$ induced by $\psi_{1}$ is surjective. Because the kernel of $\psi_{1}$ is a skyscraper sheaf, $\psi_{1}$ induces an isomorphism $\left(\pi^{*} \mathcal{E}\right)^{\sharp} \rightarrow\left(\pi^{*}\left(\pi_{*} \mathcal{V}\right)\right)^{\sharp}$. Since every step is functorial, the result follows from the canonical isomorphism $\left(\pi^{*}\left(\pi_{*} \mathcal{V}\right)\right)^{\sharp} \cong \mathcal{V}$.

Proposition 4.3. Let $\rho_{1}, \rho_{2}: \pi_{*} \mathcal{V} \rightarrow \mathcal{Q}$ be two admissible quotients and let $\mathcal{E}_{1}=\operatorname{ker} \rho_{1}, \mathcal{E}_{2}=\operatorname{ker} \rho_{2}$. Every isomorphism $u: \mathcal{E}_{1} \cong \mathcal{E}_{2}$ can be extended to an isomorphism $\psi: \pi_{*} \mathcal{V} \cong \pi_{*} \mathcal{V}$, i.e. we have a commutative diagram

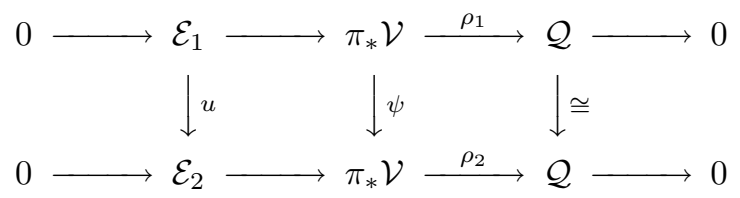

The next proposition deals with the automorphism group of $\pi_{*} \mathcal{V}$.

Proposition 4.4. Let $\mathcal{V}$ be a locally free sheaf on $\tilde{C}$. Every automorphism of $\pi_{*} \mathcal{V}$ as an $\mathcal{O}_{C}$-module can be induced from an automorphism of $\mathcal{V}$ as an $\mathcal{O}_{\tilde{C}}$-module. Hence there is a canonical isomorphism Aut $_{\mathcal{O}_{C}}\left(\pi_{*} \mathcal{V}\right) \cong A u t_{\mathcal{O}_{\tilde{C}}}(\mathcal{V})$.

Proof. Let $u: \pi_{*} \mathcal{V} \rightarrow \pi_{*} \mathcal{V}$ be an automorphism of $\pi_{*} \mathcal{V}$ as an $\mathcal{O}_{C}$-module. It induces canonically an automorphism $\bar{u}: \pi^{*} \pi_{*} \mathcal{V} \rightarrow \pi^{*} \pi_{*} \mathcal{V}$ as an $\mathcal{O}_{\tilde{C}}$-module. Let $\mathcal{T}^{\prime} \subset \pi^{*} \pi_{*} \mathcal{V}$ be the torsion part. Then $\bar{u}\left(\mathcal{T}^{\prime}\right)=\mathcal{T}^{\prime}$, and it induces an automorphism $u^{\sharp}:\left(\pi^{*} \pi_{*} \mathcal{V}\right)^{\sharp} \rightarrow\left(\pi^{*} \pi_{*} \mathcal{V}\right)^{\sharp}$. Since $\mathcal{V}$ is locally free, there is a canonical isomorphism $\left(\pi^{*} \pi_{*} \mathcal{V}\right)^{\sharp} \cong \mathcal{V}$. We obtain an automorphism $\tilde{u}: \mathcal{V} \rightarrow \mathcal{V}$ as an $\mathcal{O}_{\tilde{C}}$-module. Since every step is functorial, it establishes an isomorphism $A u t_{\mathcal{O}_{C}}\left(\pi_{*} \mathcal{V}\right) \cong A u t_{\mathcal{O}_{\tilde{C}}}(\mathcal{V})$.

Next we assume $C$ is a rational nodal curve with $n$ nodes. We describe a method to calculate $e\left(\mathfrak{M}_{C}(r, n)\right)$.

Let $\mathcal{E}$ be a stable sheaf in $\mathfrak{M}_{C}(r, n)$, and let $\mathcal{V}=\left(\pi^{*} \mathcal{E}\right)^{\sharp}$ be the torsion free part of $\pi^{*} \mathcal{E}$. Then $\mathcal{V}$ be a locally free sheaf of rank $r$ on $\tilde{C}$. Since $\tilde{C} \cong \mathbf{P}^{1}$, by Grothendieck's Lemma, $\mathcal{V} \cong \mathcal{O}\left(l_{1}\right) \oplus \mathcal{O}\left(l_{2}\right) \oplus \cdots \oplus \mathcal{O}\left(l_{r}\right)$ for some integers $l_{1} \leq l_{2} \leq \cdots \leq l_{r}$. There is a decomposition of $\mathfrak{M}_{C}(r, n)$,

$$
\mathfrak{M}_{C}(r, n)=\bigsqcup \mathfrak{M}_{a_{1}, a_{2}, \cdots, a_{n}}^{l_{1}, \cdots, l_{r}},
$$

such that $[\mathcal{E}] \in \mathfrak{M}_{a_{1}, a_{2}, \cdots, a_{n}}^{l_{1}, \cdots, l_{r}}$ if and only if

$$
\left(\pi^{*} \mathcal{E}\right)^{\sharp} \cong \mathcal{O}\left(l_{1}\right) \oplus \mathcal{O}\left(l_{2}\right) \oplus \cdots \oplus \mathcal{O}\left(l_{r}\right)
$$

and

$$
\mathcal{E}_{x_{i}} \cong \mathcal{O}_{x_{i}}^{\oplus a_{i}} \oplus m_{x_{i}}^{\oplus\left(r-a_{i}\right)} .
$$

Let $\mathcal{E}_{1}, \mathcal{E}_{2}$ be the kernels of two admissible quotients $\rho_{1}, \rho_{2}: \pi_{*} \mathcal{V} \rightarrow \mathcal{Q}$ respectively. The automorphism group of $\mathcal{Q}$ is a direct sum of automorphism groups of $\mathcal{Q}_{x_{i}}$. Let $G_{i}=\operatorname{Aut}\left(\mathcal{Q}_{i}\right)$. Then $G_{i} \cong G L\left(a_{i}, \mathbf{C}\right)$. There is an $\operatorname{Aut}(\mathcal{V}) \times \prod G_{i}$ action on $\operatorname{Hom}\left(\pi_{*} \mathcal{V}, \mathcal{Q}\right), \rho \longrightarrow g \circ \rho \circ u$, where $\rho \in \operatorname{Hom}\left(\pi_{*} \mathcal{V}, \mathcal{Q}\right), u \in \operatorname{Aut}(\mathcal{V})$ and $g \in \prod G_{i}$. Proposition 4.3 says that $\mathcal{E}_{1} \cong \mathcal{E}_{2}$ if and only if $\rho_{1}$ and $\rho_{2}$ lie in the same orbit of $\operatorname{Hom}\left(\pi_{*} \mathcal{V}, \mathcal{Q}\right)$ under this group action.

Next we work out a matrix form of these results under suitable bases. 
Let $V_{i}$ and $W_{i}$ be the fibres of $\mathcal{V}$ at $p_{i}$ and $q_{i}$ respectively. Then $\left(\pi_{*} \mathcal{V}\right) \otimes \mathbf{C}_{x_{i}} \cong$ $V_{i} \oplus W_{i}$. Since $\mathcal{Q}_{x_{i}}=\mathbf{C}^{\oplus a_{i}}$, every homomorphism $\rho: \pi_{*} \mathcal{V} \rightarrow \mathcal{Q}$ gives an element in the vector space

$$
U=\oplus_{i=1}^{n}\left(\operatorname{Hom}\left(V_{i}, \mathbf{C}^{\oplus a_{i}}\right) \oplus \operatorname{Hom}\left(W_{i}, \mathbf{C}^{\oplus a_{i}}\right)\right) .
$$

Fix an isomorphism $\mathcal{V} \cong \mathcal{O}\left(l_{1}\right) \oplus \mathcal{O}\left(l_{2}\right) \oplus \cdots \oplus \mathcal{O}\left(l_{r}\right)$ once and for all. For any summand $\mathcal{O}\left(l_{i}\right)$, there is an isomorphism of stalks $\mathcal{O}\left(l_{i}\right)_{x} \cong \mathcal{O}_{x}$ by the locally freeness of $\mathcal{O}\left(l_{i}\right)$. Those isomorphisms at $p_{i}$ and $q_{i}$ give rise to bases $e_{i}^{k} \in V_{i}$ and $f_{i}^{k} \in W_{i}$. Fix all these choices once and for all. Now an element $\rho \in U$ corresponds to a set of $a_{i} \times r$ matrixes

$$
\left\{A_{i}, B_{i}\right\}_{i=1,2, \cdots, n} .
$$

Let $\rho_{i}^{\prime} \in \operatorname{Hom}\left(V_{i}, \mathbf{C}^{\oplus a_{i}}\right), \rho_{i}^{\prime \prime} \in \operatorname{Hom}\left(W_{i}, \mathbf{C}^{\oplus a_{i}}\right)$ and $v_{i}=\left(v_{i}^{1}, v_{i}^{2}, \cdots, v_{i}^{r}\right)^{t} \in V_{i}, w_{i}=$ $\left(w_{i}^{1}, w_{i}^{2}, \cdots, w_{i}^{r}\right)^{t} \in W_{i}$. Then

$$
\rho_{i}^{\prime}\left(v_{i}\right)=A_{i}\left(\begin{array}{c}
v_{i}^{1} \\
v_{i}^{2} \\
\cdots \\
v_{i}^{r}
\end{array}\right), \rho_{i}^{\prime \prime}\left(w_{i}\right)=B_{i}\left(\begin{array}{c}
w_{i}^{1} \\
w_{i}^{2} \\
\ldots \\
w_{i}^{r}
\end{array}\right) .
$$

Corollary 4.5. A quotient $\left\{A_{i}, B_{i}\right\}$ is admissible if and only if the ranks of $A_{i}$ and $B_{i}$ are both equal to $a_{i}$ for all $i$. In particular, for an admissible quotient $\left\{A_{i}, B_{i}\right\}$, one has $a_{i} \leq r$.

Proof. Follows from proposition 4.2. $\square$

Now we consider the $\operatorname{Aut}(\mathcal{V}) \times \prod G_{i}$ action on $\operatorname{Hom}\left(\pi_{*} \mathcal{V}, \mathcal{Q}\right)$.

Evaluated at a closed point $x \in \tilde{C}$, every automorphism $u \in \operatorname{Aut}(\mathcal{V})$ gives rise to an automorphism in $\operatorname{Aut}\left(V_{x}\right)$, where $V_{x}$ is the fibre of $\mathcal{V}$ at $x$. Therefore, every $u \in \operatorname{Aut}(\mathcal{V})$ gives rise to an element

$$
\prod_{i} u\left(p_{i}\right) \times \prod_{i} u\left(q_{i}\right) \in \prod_{i} \operatorname{Aut}\left(V_{i}\right) \times \prod_{i} \operatorname{Aut}\left(W_{i}\right) .
$$

Let $G^{\prime} \subset \prod_{i} A u t\left(V_{i}\right) \times \prod_{i} A u t\left(W_{i}\right)$ be the subgroup of elements derived in this way. Since $G_{i} \cong G L\left(a_{i}, \mathbf{C}\right)$, there is an $G^{\prime} \times \prod G L\left(a_{i}, \mathbf{C}\right)$ action on the vector space $U$ of all quotients $\left\{A_{i}, B_{i}\right\}_{i=1,2, \cdots, n}$, which is given by

$$
\left\{A_{i}, B_{i}\right\} \longrightarrow\left\{g_{i} A_{i} u\left(p_{i}\right), g_{i} B_{i} u\left(q_{i}\right)\right\},
$$

where $\prod u\left(p_{i}\right) \times \prod u\left(q_{i}\right) \in G^{\prime}$ and $g_{i} \in G L\left(a_{i}, \mathbf{C}\right)$. Two quotients $\left\{A_{i}, B_{i}\right\}$ and $\left\{A_{i}^{\prime}, B_{i}^{\prime}\right\}$ are equivalent if they lie in one and the same orbit under this group action.

An application of this formulation is to determine whether $\mathcal{E} \otimes \mathcal{L} \cong \mathcal{E}$ for $\mathcal{L} \in$ $\operatorname{Pic}^{0} C$.

Let $\rho: \pi_{*} \mathcal{V} \rightarrow \mathcal{Q}$ be an admissible quotient with corresponding matrixes $\left\{A_{i}, B_{i}\right\}$ and let $\mathcal{E}=\operatorname{ker} \rho$. Let $\mathcal{L} \in \operatorname{Pic}^{0} C$ be given by the matrixes $\left\{1, t_{i}\right\}$, where $t_{i} \in \mathbf{C}^{*}$. The exact sequence

$$
0 \longrightarrow \mathcal{E} \longrightarrow \pi_{*} \mathcal{V} \stackrel{\rho}{\longrightarrow} \mathcal{Q} \longrightarrow 0
$$

induces an exact sequence

$$
0 \longrightarrow \mathcal{E} \otimes \mathcal{L} \longrightarrow \pi_{*} \mathcal{V} \otimes \mathcal{L} \stackrel{\rho \otimes 1}{\longrightarrow} \mathcal{Q} \otimes \mathcal{L} \longrightarrow 0 .
$$


Note that $\pi_{*} \mathcal{V} \otimes \mathcal{L} \cong \pi_{*} \mathcal{V}$ and the quotient $\rho \otimes 1: \pi_{*} \mathcal{V} \otimes \mathcal{L} \rightarrow \mathcal{Q} \otimes \mathcal{L}$ is also admissible. Fix an isomorphism $\mathcal{Q} \otimes \mathcal{L} \cong \mathcal{Q}$ and choose corresponding bases, $\rho \otimes 1$ is given by the matrixes $\left\{A_{i}, t_{i} B_{i}\right\}$.

We are now ready to calculate the Euler number $e\left(\mathfrak{M}_{C}(r, n)\right)$. For the purpose of this paper, we only consider the case $r=2$ and $n=1$.

Proposition 4.6. Let $\mathfrak{M}_{a_{1}, a_{2}, \cdots, a_{n}}^{l_{1}, l_{2}}$ be a stratum in $\mathfrak{M}_{C}(2,1)$ such that $\sum a_{i} \geq 2$. Then $e\left(\mathfrak{M}_{a_{1}, a_{2}, \cdots, a_{n}}^{l_{1}, l_{2}}\right)=0$.

Proof. For simplicity, we consider only the stratum $\mathfrak{M}_{1,1,0, \cdots, 0}^{l_{1}, l_{2}}$ as illustration. Let $[\mathcal{E}] \in \mathfrak{M}_{1,1,0, \cdots, 0}^{l_{1}, l_{2}}$ be the kernel of an admissible quotient $\left\{A_{i}, B_{i}\right\}$. We can choose a suitable base such that $A_{1}=A_{2}=(1,0), B_{1}=B_{2}=(0,1)$. For an odd prime $p$, let $\mathcal{L}$ be given by $t_{1}=1, t_{2}=\zeta$, where $\zeta$ is a $p$-th primitive root of unity. Then $\mathcal{L}^{\otimes p}=\mathcal{O}_{C}$ and $\mathcal{E} \otimes \mathcal{L}$ corresponds to the quotient $\left\{A_{i}, t_{i} B_{i}\right\}$. It is direct to verify that $\left\{A_{i}, t_{i} B_{i}\right\}$ and $\left\{A_{i}, B_{i}\right\}$ are not equivalent, hence $\mathcal{E} \otimes \mathcal{L}$ and $\mathcal{E}$ are not isomorphic. So we get a free $\mathbf{Z} /(p)$ action on $\mathfrak{M}_{1,1,0, \cdots, 0}^{l_{1}, l_{2}}$. Because $p$ can be chose arbitrarily large, $e\left(\mathfrak{M}_{1,1,0, \cdots, 0}^{l_{1}, l_{2}}\right)=0$.

Since $\mathfrak{M}_{0,0, \cdots, 0}^{l_{1}, l_{2}}$ is empty, by this proposition, the contribution to the Euler number $e\left(\mathfrak{M}_{C}(2,1)\right)$ comes from strata $\mathfrak{M}_{a_{1}, a_{2}, \cdots, a_{n}}^{l_{1}, l_{2}}$ with $\sum a_{i}=1$. Because $\chi(\mathcal{E})=1$ and $\mathcal{E}$ fits into an exact sequence

$$
0 \longrightarrow \mathcal{E} \longrightarrow \pi_{*}\left(\mathcal{O}\left(l_{1}\right) \oplus \mathcal{O}\left(l_{2}\right)\right) \longrightarrow \mathbf{C}_{x_{i}} \longrightarrow 0,
$$

the stability of $\mathcal{E}$ forces $l_{1}=l_{2}=0$. Every $\mathfrak{M}_{0, \cdots, 1, \cdots, 0}^{0,0}$ is a set of a single point. Therefore,

Proposition 4.7. The Euler number $e\left(\mathfrak{M}_{C}(2,1)\right)$ is equal to $n$, which is the number of nodes on $C$.

Let $D=2 C_{0}$ be a divisor in the set $\mathcal{W}_{3}^{0}$. Then $\mathfrak{M}_{D}^{1}$ is isomorphic to $\mathfrak{M}_{C_{0}}(2,1)$. The number of nodes on $C_{0}$ is equal to the arithmetic genus $g=\frac{1}{2} H^{2}+1$ of $C_{0}$. Therefore,

Proposition 4.8. e $\left(\mathfrak{M}_{D}^{1}\right)=g$.

5. Calculation of $e\left(\Phi^{-1}\left(\mathcal{W}_{3}^{0}\right)\right)$, Part II. This is the second part of the calculation of $e\left(\Phi^{-1}\left(\mathcal{W}_{3}^{0}\right)\right)$. As we mentioned in the previous section, $\mathfrak{M}_{D}$ is a disjoint union of $\mathfrak{M}_{D}^{1}$ and $\mathfrak{M}_{D}^{2}$ for $D \in \mathcal{W}_{3}^{0}$. We have calculated $e\left(\mathfrak{M}_{D}^{1}\right)$. In this section, we will show that $e\left(\mathfrak{M}_{D}^{2}\right)=0$.

Let $C_{0} \subset S$ be a nodal curve, and let $C$ be the associated nonreduced curve to the divisor $2 C_{0}$. Let $p$ be a node on $C_{0}$, and $\pi_{0}: \hat{C}_{0} \rightarrow C_{0}$ be the partial normalization of $C_{0}$ at $p$. Now we construct a curve $\hat{C}$, which is an infinitesimal extension of $\hat{C}_{0}$, and a finite morphism $\pi: \hat{C} \rightarrow C$ called a partial normalization of $C$.

We pick a small neighborhood $U$ around $p$ on the surface $S$, such that $C$ is defined by $x^{2} y^{2}=0$ in $U$. Let $\mathbf{C}\{x, y\}$ be the ring of holomorphic functions on $U$. Then $\mathcal{O}_{C}(U \cap C)=\mathbf{C}\{x, y\} /\left(x^{2} y^{2}\right)$. The injective homomorphism

$$
\psi: \mathbf{C}\{x, y\} /\left(x^{2} y^{2}\right) \rightarrow \mathbf{C}\{x, u\} /\left(u^{2}\right) \oplus \mathbf{C}\{v, y\} /\left(v^{2}\right)
$$

is a local isomorphism except at $p$. Remove the point $p$ on $C$ and glue the pieces defined by the ringed space $\mathbf{C}\{x, u\} /\left(u^{2}\right) \oplus \mathbf{C}\{v, y\} /\left(v^{2}\right)$ along $\psi$, we get a curve $\hat{C}$, and a finite map $\pi: \hat{C} \rightarrow C$. There is a canonical exact sequence

$$
0 \longrightarrow \mathcal{O}_{C} \longrightarrow \pi_{*} \mathcal{O}_{\hat{C}} \longrightarrow \mathcal{A} \longrightarrow 0,
$$


where $\mathcal{A} \cong \mathbb{C}[x, y] /\left(x^{2}, y^{2}\right)$ is a skyscraper sheaf supported at $p$. Moreover, there is a commutative diagram

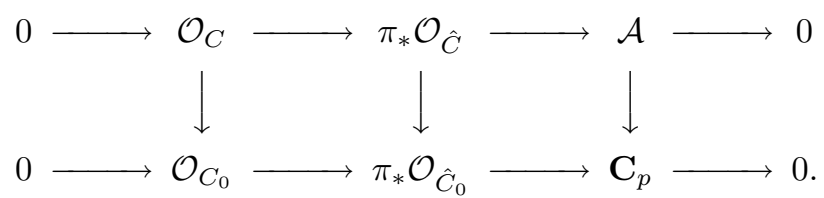

Let $\mathcal{I}$ and $\hat{\mathcal{I}}$ be the nilpotent ideal sheaves of $\mathcal{O}_{C}$ and $\mathcal{O}_{\hat{C}}$ respectively. Then $\chi(\hat{\mathcal{I}})=\chi(\mathcal{I})+3$. It implies that $\operatorname{deg} \hat{\mathcal{I}}=\operatorname{deg} \mathcal{I}+2$. Let $C$ be a rational nodal curve on a $K 3$ surface and let $\tilde{C} \rightarrow C$ be the normalization of $C$. Then $\operatorname{deg} \mathcal{I}=2-2 g=H^{2}$. Because the number of nodes on $C$ is equal to $g, \operatorname{deg} \tilde{\mathcal{I}}=\operatorname{deg} \mathcal{I}+2 g=2$.

Proposition 5.1. Let $C$ be a nonreduced curve with nilpotent ideal sheaf $\mathcal{I}$. Suppose $\mathcal{I}$ is invertible as a sheaf of $\mathcal{O}_{C_{0}}$-modules and $\operatorname{deg} \mathcal{I}>0$. Let $\mathcal{E}$ be a pure sheaf of $\mathcal{O}_{C}$-modules such that $\mathcal{E}_{\eta} \cong \mathcal{O}_{\eta}$ at the generic point $\eta$ of $C$. Then $\mathcal{E}$ is not stable.

Proof. Let $\mathcal{E}$ be such a sheaf and let $\mathcal{E}_{0}^{\sharp}$ be the torsion free part of $\mathcal{E}_{0}=\mathcal{E} \otimes \mathcal{O}_{C_{0}}$, considered as a sheaf of $\mathcal{O}_{C_{0}}$-modules. There is a canonical homomorphism $\mathcal{E} \rightarrow \mathcal{E}_{0}^{\sharp}$. Every quotient $\mathcal{E} \rightarrow \mathcal{F}$ with $\mathcal{F}$ a torsion free $\mathcal{O}_{C_{0}}$-module is equivalent to $\mathcal{E} \rightarrow \mathcal{E}_{0}^{\sharp}$. Therefore, for the stability of $\mathcal{E}$, it is enough to check the quotient $\mathcal{E} \rightarrow \mathcal{E}_{0}^{\sharp}$.

We start with the exact sequence

$$
0 \longrightarrow \mathcal{I} \longrightarrow \mathcal{O}_{C} \longrightarrow \mathcal{O}_{C_{0}} \longrightarrow 0 .
$$

Tensoring with $\mathcal{E}$, we obtain

$$
\mathcal{E}_{0} \otimes \mathcal{I} \longrightarrow \mathcal{E} \longrightarrow \mathcal{E}_{0} \longrightarrow 0
$$

Let $\mathcal{T}^{\prime}$ be the torsion part of $\mathcal{E}_{0} \otimes \mathcal{I}$, and let $\left(\mathcal{E}_{0} \otimes \mathcal{I}\right)^{\sharp}=\left(\mathcal{E}_{0} \otimes \mathcal{I}\right) / \mathcal{T}^{\prime}$. There is an exact sequence

$$
0 \longrightarrow\left(\mathcal{E}_{0} \otimes \mathcal{I}\right)^{\sharp} \longrightarrow \mathcal{E} \longrightarrow \mathcal{E}_{0} \longrightarrow 0 .
$$

On the other hand, we have

$$
0 \longrightarrow \mathcal{K} \longrightarrow \mathcal{E} \longrightarrow \mathcal{E}_{0}^{\sharp} \longrightarrow 0
$$

Because $\mathcal{I}$ is an invertible sheaf of $\mathcal{O}_{C_{0}}$-modules, the torsion part of $\mathcal{E}_{0}$ is isomorphic to $\mathcal{T}^{\prime} . \chi(\mathcal{K})=\chi(\mathcal{E})-\chi\left(\mathcal{E}_{0}^{\sharp}\right)=\chi\left(\mathcal{E}_{0} \otimes \mathcal{I}\right)$. Because $\chi\left(\mathcal{E}_{0} \otimes \mathcal{I}\right)=\chi\left(\mathcal{E}_{0}\right)+\operatorname{deg} \mathcal{I}>$ $\chi\left(\mathcal{E}_{0}\right)>\chi\left(\mathcal{E}_{0}^{\sharp}\right), \mathcal{E}$ is not stable.

Let $\pi: \hat{C} \rightarrow C$ be the partial normalization of $C$ at $p$. Let $\mathcal{F}$ be a sheaf of $\mathcal{O}_{C}$-modules which is pure of dimension 1 . Then there is a canonical homomorphism $\mathcal{F} \rightarrow \pi_{*}\left(\pi^{*} \mathcal{F}\right)$. Let $T_{0} \subset \pi^{*} \mathcal{F}$ be the maximal subsheaf of dimension 0 , we get a sheaf $\left(\pi^{*} \mathcal{F}\right)^{\sharp}=\pi^{*} \mathcal{F} / \mathcal{T}_{0}$ which is pure of dimension 1 , and there is a canonical injective homomorphism $\mathcal{F} \rightarrow \pi_{*}\left(\pi^{*} \mathcal{F}\right)^{\sharp}$. The cokernel $\mathcal{T}$ is a skyscraper sheaf supported at $p$. Note that if $\mathcal{F}$ satisfies $\mathcal{I} \mathcal{F} \neq 0$, so does $\left(\pi^{*} \mathcal{F}\right)^{\sharp}$ as a sheaf of $\mathcal{O}_{\hat{C}}$-modules.

The notion of admissible quotients can be defined in the same way as in section 4 , and propositions 4.2-4.4 are also true in this case.

Let $\rho: \pi_{*} \mathcal{E} \rightarrow \mathcal{Q}$ be an admissible quotient, and let $p$ be a node on $C$ with $\pi^{-1}(p)=\left\{q_{1}, q_{2}\right\}$. Then $\left(\pi_{*} \mathcal{E}\right)_{p}=\mathcal{E}_{q_{1}} \oplus \mathcal{E}_{q_{2}}$. We let $\iota_{i}: \mathcal{E}_{q_{i}} \rightarrow\left(\pi_{*} \mathcal{E}\right)_{p}$ and $p_{i}:$ 
$\left(\pi_{*} \mathcal{E}\right)_{p} \rightarrow \mathcal{E}_{q_{i}}$ be the natural injections and projections respectively. Define $\rho^{i}$ as compositions

$$
\rho^{i}: \mathcal{E}_{q_{i}} \stackrel{\iota_{i}}{\rightarrow}\left(\pi_{*} \mathcal{E}\right)_{p} \stackrel{\rho}{\rightarrow} \mathcal{Q}_{p}
$$

Because $\rho$ is admissible, $\rho^{i}$ are both surjective homomorphisms. Clearly $\rho=$ $\rho^{1} p_{1}+\rho^{2} p_{2}$. For $t \in \mathbf{C}^{*}$, we define $\rho_{t}=\rho^{1} p_{1}+t \rho^{2} p_{2}$. It gives rise to a surjective homomorphism $\rho_{t}: \pi_{*} \mathcal{E} \rightarrow \mathcal{Q}$ which is also admissible.

Let $\pi: \hat{C} \rightarrow C$ be the partial normalization of $C$ at $p$. Apply the above construction to the canonical exact sequence

$$
0 \longrightarrow \mathcal{O}_{C} \longrightarrow \pi_{*} \mathcal{O}_{\hat{C}} \stackrel{\rho}{\longrightarrow} \mathcal{A} \longrightarrow 0,
$$

and let $\mathcal{L}_{t}=\operatorname{ker} \rho_{t}$. Then $\mathcal{L}_{t}$ is invertible for every $t \in \mathbf{C}^{*}$. The set of these invertible sheaves form a subgroup $G_{p} \subset \operatorname{Pic}^{0} C$. Clearly $G_{p} \cong \mathbf{C}^{*}$.

For any admissible quotient $\rho: \pi_{*} \mathcal{E} \rightarrow \mathcal{Q}$, let $\mathcal{K}_{t}$ be the kernel of $\rho_{t}$.

Lemma 5.2. There is a canonical isomorphism between $\mathcal{L}_{t} \otimes \mathcal{K}_{s}$ and $\mathcal{K}_{s t}$.

Now we give a decomposition on $\mathfrak{M}_{D}^{2}$ for $D \in \mathcal{W}_{3}^{0}$. Let $\pi: \tilde{C} \rightarrow C$ be the normalization of $C$. Let $\mathfrak{M}_{\tilde{\mathcal{F}}, \mathcal{T}}$ be the subset consists of stable sheaves $\mathcal{F}$ such that $\left(\pi^{*} \mathcal{F}\right)^{\sharp} \cong \tilde{\mathcal{F}}$ and $\pi_{*} \tilde{\mathcal{F}} / \mathcal{F} \cong \mathcal{T}$. We get a decomposition $\mathfrak{M}_{D}^{2}=\sqcup \mathfrak{M}_{\tilde{\mathcal{F}}, \mathcal{T}}$. In fact, for every nonempty stratum $\mathfrak{M}_{\tilde{\mathcal{F}}, \mathcal{T}}, \mathcal{T}$ is nonzero. Because if $\mathfrak{M}_{\tilde{\mathcal{F}}, 0}$ is nonempty, a sheaf $\mathcal{F}$ in $\mathfrak{M}_{\tilde{\mathcal{F}}, 0}$ is the direct image of a sheaf on $\tilde{C}$, i.e. $\mathcal{F} \cong \tilde{\pi}_{*} \mathcal{E}$ for a sheaf $\mathcal{E}$ of $\mathcal{O}_{\tilde{C}}$-modules. By proposition $5.1, \mathcal{E}$ is not stable, which violates the stability of $\mathcal{F}$.

Let $\mathfrak{M}_{\tilde{\mathcal{F}}, \mathcal{T}}$ be a stratum, and let $p \in C$ be a node such that $\mathcal{T}_{p} \neq 0$. There is a subgroup $G_{p} \subset \operatorname{Pic}^{0} C$ defined as above, and a $G$-action on $\mathfrak{M}_{\tilde{\mathcal{F}}, \mathcal{T}}$ defined by tensorization. Next we will show that this group action is free on $\mathfrak{M}_{\tilde{\mathcal{F}}, \mathcal{T}}$. The following lemma is useful in the proof.

Let $\mathcal{E}$ be a pure sheaf of $\mathcal{O}_{C}$-modules such that $\mathcal{E}_{\eta} \cong \mathcal{O}_{\eta}$ at the generic point $\eta$ of $C$. Let $\mathcal{E}^{\prime \prime}$ be the torsion free part of $\mathcal{E}_{0}=\mathcal{E} \otimes_{\mathcal{O}_{C}} \mathcal{O}_{C_{0}}$, and let $\mathcal{E}^{\prime}$ be the kernel of the restriction homomorphism $f: \mathcal{E} \rightarrow \mathcal{E}^{\prime \prime}$. Since $f$ is not an isomorphism, $\mathcal{E}^{\prime}$ and $\mathcal{E}^{\prime \prime}$ are both rank 1 torsion free sheaves of $\mathcal{O}_{C_{0}}$-modules whose automorphism groups are $\mathbf{C}^{*}$.

Lemma 5.3. Let $\psi: \mathcal{E} \rightarrow \mathcal{E}$ be an automorphism and let $c: \mathcal{E}^{\prime} \rightarrow \mathcal{E}^{\prime}$ and $d: \mathcal{E}^{\prime \prime} \rightarrow \mathcal{E}^{\prime \prime}$ be the induced automorphisms. Then they fit into the commutative diagram

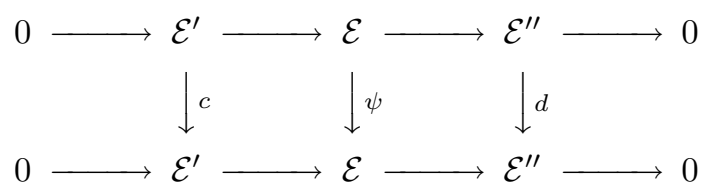

and $c=d$.

Proof. Consider $\psi^{\prime}=\psi-c \cdot i d: \mathcal{E} \rightarrow \mathcal{E}$. Clearly $\psi^{\prime}\left(\mathcal{E}^{\prime}\right)=0$. It induces a homomorphism $u: \mathcal{E}^{\prime \prime} \rightarrow \mathcal{E}$. Composed with $\mathcal{E} \rightarrow \mathcal{E}^{\prime \prime}$, we get $h: \mathcal{E}^{\prime \prime} \rightarrow \mathcal{E}^{\prime \prime}$. Since $\mathcal{E}^{\prime \prime}$ is torsion free and has rank 1 as an $\mathcal{O}_{C_{0}}$-module, $h$ is a multiplication by $(d-c)$. If $h \neq 0$, then after scaling $u$ by $\frac{1}{h}, u$ splits the exact sequence and hence $\mathcal{E} \cong \mathcal{E}^{\prime} \oplus \mathcal{E}^{\prime \prime}$, which contradicts to $\mathcal{E}_{\eta} \cong \mathcal{O}_{\eta}$. Therefore $h=0$, i.e. $c=d$. 
Proposition 5.4. Let $\mathfrak{M}_{\tilde{\mathcal{F}}, \mathcal{T}}$ be a stratum such that $\mathcal{T}_{p} \neq 0$ for a node $p \in C$. Then the associated $G_{p}$-action on $\mathfrak{M}_{\tilde{\mathcal{F}}, \mathcal{T}}$ is free. Therefore, by the decomposition of $\mathfrak{M}_{D}^{2}, e\left(\mathfrak{M}_{D}^{2}\right)=0$.

Proof. Let $\pi: \hat{C} \rightarrow C$ be the partial normalization of $C$ at $p$. Let $\mathcal{F}$ be a stable sheaf in $\mathfrak{M}_{\tilde{\mathcal{F}}, \mathcal{T}}$. Then $\mathcal{F}$ fits into the exact sequence

$$
0 \longrightarrow \mathcal{F} \longrightarrow \pi_{*}\left(\pi^{*} \mathcal{F}\right)^{\sharp} \longrightarrow \mathcal{T} \longrightarrow 0 .
$$

Let $\mathcal{E}=\left(\pi^{*} \mathcal{F}\right)^{\sharp}$. Then $\rho: \pi_{*} \mathcal{E} \longrightarrow \mathcal{T}$ is clearly an admissible quotient. We let $\mathcal{K}_{t}$ be the kernel of $\rho_{t}$. Then $\mathcal{F}=\mathcal{K}_{1}$ and $\mathcal{F} \otimes \mathcal{L}_{t}=\mathcal{K}_{t}$. Suppose $\mathcal{F} \otimes \mathcal{L}_{t} \cong \mathcal{F}$ for some $\mathcal{L}_{t} \in G_{p}$, there is a commutative diagram

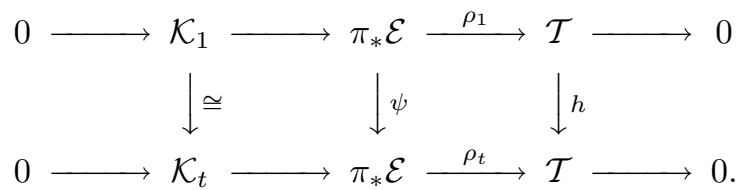

It induces the following diagram on the stalks at the node $p$,

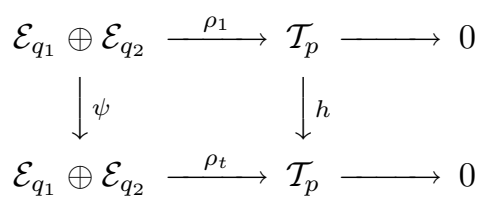

where $\left\{q_{1}, q_{2}\right\}=\pi^{-1}(p)$.

Recall that there is a canonical exact sequence for $\mathcal{E}$,

$$
0 \longrightarrow \mathcal{E}^{\prime} \longrightarrow \mathcal{E} \longrightarrow \mathcal{E}^{\prime \prime} \longrightarrow 0,
$$

where $\mathcal{E}^{\prime}$ and $\mathcal{E}^{\prime \prime}$ are nonzero torsion free sheaves of $\mathcal{O}_{C_{0}}$-modules. Let $\mathcal{T}^{i} \subset \mathcal{T}_{p}$ be the images of $\mathcal{E}_{q_{i}}^{\prime}$ under the surjective homomorphisms $\rho^{i}: \mathcal{E}_{q_{i}} \rightarrow \mathcal{T}_{p}$. Then we have the following three cases.

Case 1. $\mathcal{T}^{1} \neq 0$ and $\mathcal{T}^{2} \neq 0$.

Since $\psi: \pi_{*} \mathcal{E} \rightarrow \pi_{*} \mathcal{E}$ is induced from an automorphism of $\mathcal{E}, \psi\left(\mathcal{E}_{q_{i}}\right)=\mathcal{E}_{q_{i}}$. Consider the restriction of the diagram to $\mathcal{E}_{q_{i}}^{\prime}$ respectively, by Lemma 5.3 , there are commutative diagrams

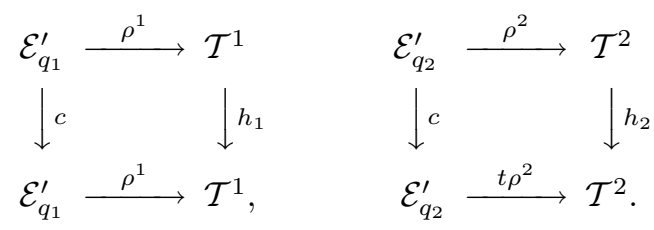

If $\mathcal{T}^{1} \cap \mathcal{T}^{2} \neq\{0\}$, let $0 \neq x \in \mathcal{T}^{1} \cap \mathcal{T}^{2}$. Then from the left diagram, $h(x)=h_{1}(x)=c x$, and from the right diagram, $h(x)=h_{2}(x)=c t x$. It implies that $t=1$ and therefore the group action is free. Next we assume $\mathcal{T}^{1} \cap \mathcal{T}^{2}=\{0\}$. Let $x \in \mathcal{T}^{1}$ be a nonzero element. Then the image $\bar{x}$ of $x$ in $\mathcal{T}_{p} / \mathcal{T}^{2}$ is nonzero. From the commutative diagram

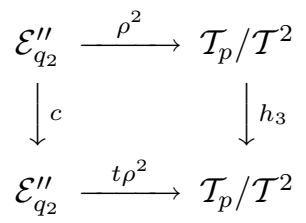


we have $h_{3}(\bar{x})=c t \bar{x}$. Because $h(x)=h_{1}(x)=c x, h_{3}(\bar{x})=c \bar{x}$. It implies that $t=1$.

Case 2. $\mathcal{T}^{1} \neq 0$ and $\mathcal{T}^{2}=0$ (Or equivalently $\mathcal{T}^{1}=0$ and $\left.\mathcal{T}^{2} \neq 0\right)$.

Since $\mathcal{T}^{2}=0$ and $\mathcal{E}_{q_{2}} \stackrel{\rho^{2}}{\rightarrow} \mathcal{T}_{p}$ is surjective, there is a surjective morphism $\rho^{2}: \mathcal{E}_{q_{2}}^{\prime \prime} \rightarrow$ $\mathcal{T}_{p}$ and a commutative diagram

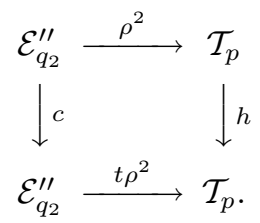

Let $0 \neq x \in \mathcal{T}^{1}$. We already have $h(x)=h_{1}(x)=c x$. However, from the above diagram, $h(x)=$ ctx. Hence $t=1$.

Case $3 . \mathcal{T}^{1}=0$ and $\mathcal{T}^{2}=0$.

Since $\mathcal{T}^{1}=0$ and $\mathcal{T}^{2}=0$, we get commutative diagrams

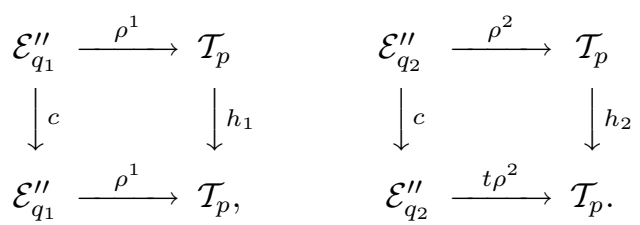

Apply the same argument as in case 1 , we get $t=1$.

Because the cardinality of the finite set $\mathcal{W}_{3}^{0}$ is $G_{g}$, combine proposition 4.8 and 5.4 , we conclude

Proposition 5.5. $e\left(\Phi^{-1}\left(\mathcal{W}_{3}^{0}\right)\right)=g G_{g}$.

\section{REFERENCES}

[1] A. Beauville, Counting rational curves on K3 surfaces, Duke Math. J., 97 (1999), pp. 99-108.

[2] J. Bryan, N. Leung, The enumerative geometry of K3 surfaces and modular forms, J. Amer. Math. Soc., 13 (2000), pp. 371-410.

[3] X. Chen, A simple proof that rational curves on K3 are nodal, Math. Ann., 324 (2002),pp. 71104.

[4] X. Chen, private communication.

[5] B. Fantechi, L. Göttsche, D. van Straten, Euler number of the compactified Jacobian and multiplicity of rational curves, J. Algebraic Geom., 8 (1999), pp. 115-133.

[6] A. Gathmann, The number of plane conics 5-fold tangent to a given curve, preprint, math.AG/0202002.

[7] R. Hartshorne, Algebraic Geometry, Springer Verlag. 1977.

[8] D. Huybrechts, M. Lehn, The geometry of moduli spaces of sheaves, Vieweg. 1997.

[9] J. Lee And N. C. Leung, Yau-Zaslow formula on $K 3$ surfaces for non-primitive classes, Geom. Topol., 9 (2005), pp. 1977-2012.

[10] J. Lee And N. C. Leung, Counting elliptic curves in K3 surfaces, J. Algebraic Geom., 15 (2006), pp. 591-601.

[11] J. LI, A note on enumerating rational curves in a K3 surface, Geometry and nonlinear partial differential equations, 53-62, AMS/IP Stud. Adv. Math. 29, 2002.

[12] J. Li and B. Wu, Note on a conjecture of Gopakumar-Vafa, Chinese Ann. Math. Ser. B, 27 (2006), pp. 219-242.

[13] S. MukaI, Symplectic structure of the moduli space of sheaves on an abelian or K3 surface, Invent. Math., 77 (1984), pp. 101-116.

[14] C. S. Seshadri, Fibrés vectoriels sur les courbes algébriques, Asterisque, 96 (1982).

[15] C. T. Simpson, Moduli of representations of the fundamental group of a smooth projective variety I, Publ. Math. IHES., 79 (1994), pp. 47-129. 
[16] T. TEOdorescu, Semistable torsion-free sheaves over curves of arithmetic genus one, $\mathrm{PhD}$ thesis. Columbia University, 1999.

[17] B. Wu, Euler number of the moduli space of sheaves on a rational nodal curve, Proc. Amer. Math. Soc., 132 (2004), pp. 1925-1936.

[18] S. T. YAU, E. ZASLOW, BPS states, string duality, and nodal curves on K3, Nucl. Phys. B, 471(1996), pp. 503-512.

[19] K. YoshiokA, Euler characteristics of SU(2) instanton moduli spaces on rational elliptic surfaces, Comm. Math. Phys., 205 (1999), pp. 501-517. 